\title{
A Mass-Flux Scheme View of a High-Resolution Simulation of a Transition from Shallow to Deep Cumulus Convection
}

\author{
ZHIMING KUANG* \\ Division of Geological and Planetary Sciences, California Institute of Technology, Pasadena, California \\ CHRISTOPHER S. BRETHERTON \\ Department of Atmospheric Sciences, University of Washington, Seattle, Washington
}

(Manuscript received 11 April 2005, in final form 11 November 2005)

\begin{abstract}
In this paper, an idealized, high-resolution simulation of a gradually forced transition from shallow, nonprecipitating to deep, precipitating cumulus convection is described; how the cloud and transport statistics evolve as the convection deepens is explored; and the collected statistics are used to evaluate assumptions in current cumulus schemes. The statistical analysis methodologies that are used do not require tracing the history of individual clouds or air parcels; instead they rely on probing the ensemble characteristics of cumulus convection in the large model dataset. They appear to be an attractive way for analyzing outputs from cloud-resolving numerical experiments. Throughout the simulation, it is found that 1) the initial thermodynamic properties of the updrafts at the cloud base have rather tight distributions; 2) contrary to the assumption made in many cumulus schemes, nearly undiluted air parcels are too infrequent to be relevant to any stage of the simulated convection; and 3) a simple model with a spectrum of entraining plumes appears to reproduce most features of the cloudy updrafts, but significantly overpredicts the mass flux as the updrafts approach their levels of zero buoyancy. A buoyancy-sorting model was suggested as a potential remedy. The organized circulations of cold pools seem to create clouds with larger-sized bases and may correspondingly contribute to their smaller lateral entrainment rates. Our results do not support a mass-flux closure based solely on convective available potential energy (CAPE), and are in general agreement with a convective inhibition (CIN)-based closure. The general similarity in the ensemble characteristics of shallow and deep convection and the continuous evolution of the thermodynamic structure during the transition provide justification for developing a single unified cumulus parameterization that encompasses both shallow and deep convection.
\end{abstract}

\section{Introduction}

Parameterizations of cumulus convection in largescale models currently employ a wide variety of assumptions about how the cumulus cloud ensemble and associated fluxes of heat, moisture, and momentum relate to large-scale variables. Many of these assumptions have not yet been adequately evaluated. Such evalua-

\footnotetext{
* Current affiliation: Department of Earth and Planetary Sciences, and Division of Engineering and Applied Sciences, Harvard University, Cambridge, Massachusetts.

Corresponding author address: Zhiming Kuang, Dept. of Earth and Planetary Sciences, Harvard University, 20 Oxford St., Cambridge, MA 02138.

E-mail: kuang@fas.harvard.edu
}

tions are difficult to make because they require data with a level of detail and accuracy that is very difficult to obtain from observations. In recent years, cloudresolving models [CRMs; here defined to include largeeddy-resolving two-dimensional (2D) as well as threedimensional (3D) models] have emerged as an alternative source of information.

For shallow (by which we mean almost nonprecipitating) cumulus convection, studies by Siebesma and Cuijpers (1995), Siebesma et al. (2003), Zhao and Austin $(2005 a, b)$, and others compared CRM results with mass-flux schemes, the most widely used type of cumulus parameterizations. The former two papers compared vertical moisture and heat-flux profiles from a CRM with predictions of a single bulk entrainingdetraining plume model, and diagnosed optimal fractional entrainment and detrainment rates for such a 
model. The latter two papers examined turbulent transport and mixing processes in six simulated shallow cumuli of different sizes, demonstrating the importance of buoyancy-sorting processes (Raymond and Blyth 1986) and of all phases of the cumulus life cycle.

CRM studies of individual cumulus congestus clouds and deep (significantly precipitating) cumulus clouds by Carpenter et al. (1998) and Cohen (2000) reached similar conclusions to Zhao and Austin. There have also been CRM studies aimed at evaluating parameterization schemes for deep cumulus convection (Lin 1999: Lin and Arakawa 1997), though these studies were limited to a 2D geometry and a coarse numerical resolution. Lin and Arakawa (1997) showed that if clouds were categorized by their maximum cloud-top height as determined by trajectory analysis, an entraining plume model similar to that used in the parameterization of Arakawa and Schubert (1974) could predict many of the cloud properties. Lin (1999) used back trajectories to examine the properties of air entering the base of the cumuli.

Several key issues for mass-flux cumulus parameterization have not been adequately addressed by prior CRM studies. These include mass-flux closure (what regulates the overall cloud-base mass flux) and partitioning (how the cumulus mass flux at each level is partitioned between different mixtures of cloud base and environmental air). Furthermore, many large-scale models use separate parameterizations for shallow and deep cumulus convection. This is most justifiable if transitions between the two are discrete, as might occur in continental deep cumulus convection in highly conditionally unstable environments. But is such a transition still discrete when the cloud ensemble is produced by a more gradually evolving forcing, such as might occur over a warm ocean?

In this study, we describe an idealized, highresolution CRM simulation of a gradually forced transition from shallow, nonprecipitating to deep, precipitating cumulus convection; explore how the cloud and transport statistics evolve as the convection deepens; and use the collected statistics to evaluate assumptions in current cumulus schemes, including cloud-base properties, the utility of an entraining plume perspective, and the mass-flux closure and partitioning problems. We compare the statistical character of shallow and deep convection in order to gain new insights into whether and how unified schemes should be developed to handle both shallow and deep convection. Our study differs from previous ones in that we use a significantly higher resolution and a 3D geometry so that we can better resolve turbulent mixing. We have also devised convenient new ways to analyze the simulated cloud ensemble, which lead to more direct evaluation of cumulus schemes and do not require tracing the history of individual clouds or air parcels.

One of our goals is to examine the utility of an entraining plume model of mixing in cumulus clouds as an organizing principle for understanding the statistical distribution of buoyancy, vertical velocity, and liquid water content as functions of height. In framing this goal, we do not imply that individual cumuli act as entraining plumes. The above-referenced CRM studies show that individual clouds are made up of a complex and time-varying spectrum of mixtures of cloud base and environmental air from many levels. Each cloud during each phase of its life cycle may therefore be regarded as contributing to many plumes of many entrainment rates in a cumulus parameterization using an entraining plume ensemble formulation, for example, see Arakawa and Schubert (1974). One should understand the analogy more in terms of fluid parcels within cumulus clouds, each undergoing a mixing history that can be roughly idealized in an ensemble sense as behaving somewhat like an entraining plume.

Section 2 contains a brief description of the model used in this study and an overview of the simulation. Analyses of the model output and implications for cumulus schemes are presented in section 3, followed by a brief summary (section 4).

\section{Model and an overview of the simulation}

We use the System for Atmospheric Modeling (SAM), which is an updated version of the Colorado State University Large-Eddy Simulation/CloudResolving Model (Khairoutdinov and Randall 2003). The model uses the anelastic equations of motion with bulk microphysics. Its prognostic thermodynamic variables are the liquid-ice static energy $s_{l i}=c_{p} T+g z-$ $L\left(q_{n}+q_{p}\right)-L_{f}\left(q_{i}+q_{s}+q_{g}\right)$, the total (nonprecipitating) water specific humidity ${ }^{1} q_{t}=q_{v}+q_{n}$ and the total precipitating water specific humidity $q_{p}$. Here $T$ is temperature, $z$ is height, $g$ is the gravitational acceleration, $c_{p}$ is isobaric specific heat, $L$ and $L_{f}$ are the latent heats of vaporization and freezing, and $q_{n}=q_{l}+q_{i}$ is the nonprecipitating condensate specific humidity, which is the sum of the specific humidities of cloud water $q_{l}$ and cloud ice $q_{i}$. In addition, $q_{v}$ is water vapor specific humidity, and $q_{s}$ and $q_{g}$ are the specific humidity of the ice phase precipitating water, snow and grau-

\footnotetext{
${ }^{1}$ While mixing ratios were used in the original description of SAM (Khairoutdinov and Randall, 2003), the actual model uses specific humidity (M. Khairoutdinov 2005, personal communication).
} 
(a) Surface heat flux

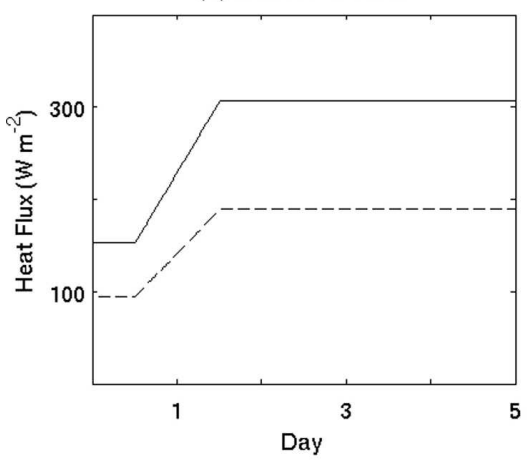

(c) $T(K)$ and $q_{v}(g / k g)$

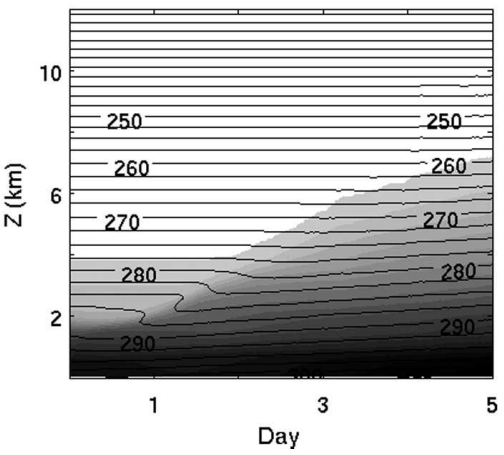

(b) Precipitation
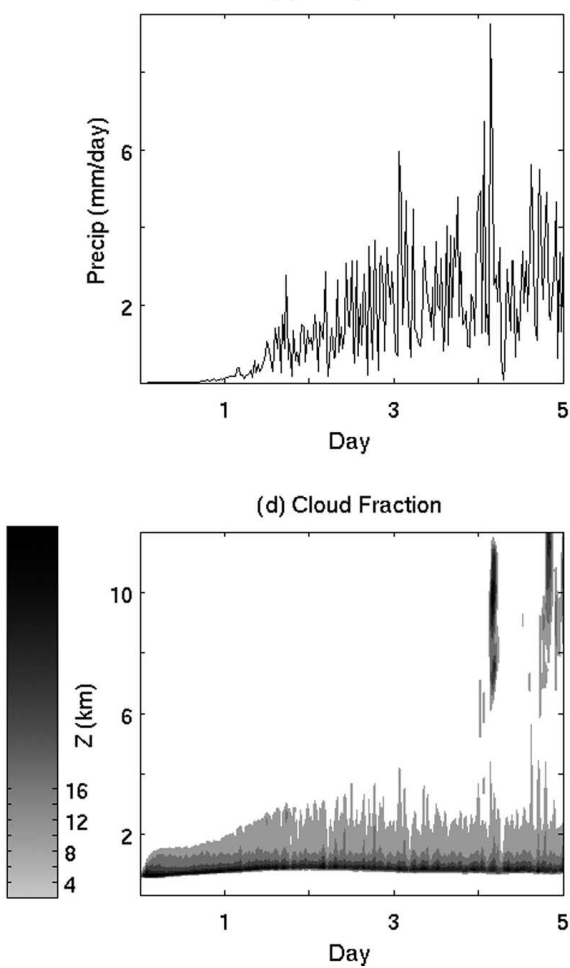

(d) Cloud Fraction

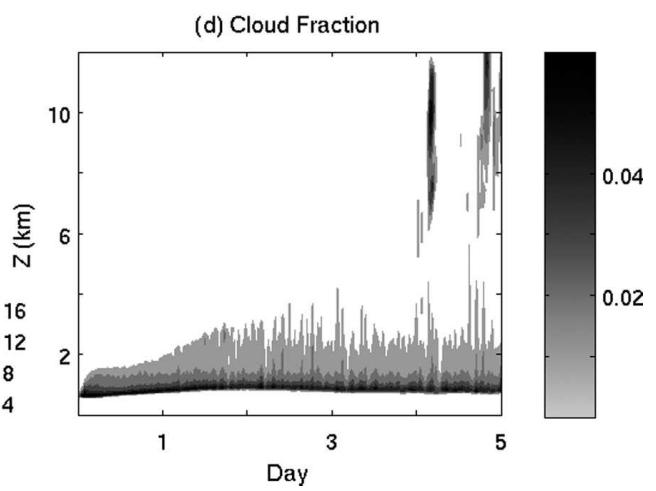

FIG. 1. (a) Surface latent heat (solid) and 10 times the sensible heat (dashed) fluxes applied in the experiment, and the evolution of domain-mean (b) precipitation, and profiles of (c) temperature (contours) and water vapor specific humidity (shading), and (d) cloud fraction.

pel, respectively. The readers are referred to Khairoutdinov and Randall (2003) for further details about the model. For this study, we use a simple Smagorinskytype scheme to represent the effect of subgrid-scale turbulence.

We use a doubly periodic domain with the model top placed at $19 \mathrm{~km}$. There are 256 grid points in the vertical, and the vertical grid size is uniformly $50 \mathrm{~m}$ below $12 \mathrm{~km}$ and gradually increases above that. A waveabsorbing layer is placed in the upper third of the domain. We use a relatively small horizontal grid size of $100 \mathrm{~m}$ in order to resolve the mixing processes between cumulus clouds of all sizes and their environment, a key aspect of the present study. This resolution is similar to that of a recent CRM intercomparison study of shallow cumulus convection (Siebesma et al. 2003). Computational constraints mandate the use of a fairly small domain size $(19.2 \mathrm{~km} \times 19.2 \mathrm{~km})$. While this domain size is insufficient to correctly simulate the long-term behavior of fully developed deep convective systems (such as in radiative-convective equilibrium simulations), it appears sufficient to accommodate the transition from shallow to deep cumulus convection, and is adequate for examining the evolution of cloud and transport statistics during this transition.
Our simulation starts from a classic and well-studied oceanic trade cumulus case derived from observations taken during the Barbados Oceanography and Meteorology Experiment (BOMEX; Holland and Rasmusson 1973; Nitta and Esbensen 1974). We use the initial sounding, surface heat and moisture fluxes, horizontalmean advective forcings, subsidence velocity, and constant, cloud-independent radiative forcings specified in the recent CRM intercomparison study of this case by Siebesma et al. (2003). Unlike Siebesma et al. (2003), we start with no horizontal winds, apply no Coriolis force, and do not nudge the mean horizontal winds at later times. These forcings allow a nearly statistically steady trade cumulus cloud ensemble to develop within 2-3 h. The forcings all decrease linearly with height above $1500 \mathrm{~m}$ and are zero at all heights above $2.5 \mathrm{~km}$. This is artificial, but is a nice simplification for our purposes since above $2.5 \mathrm{~km}$, we can assume that moist convection will be the only source of moisture and heat. After $12 \mathrm{~h}$ of simulation, we slowly ramp up the surface latent and sensible heat fluxes (Fig. 1a), with the Bowen ratio kept constant. Note that the dashed line in Fig. 1a is the sensible heat flux scaled up by a factor of 10 .

An overview of the domain mean precipitation and profiles of potential temperature, total water content, 


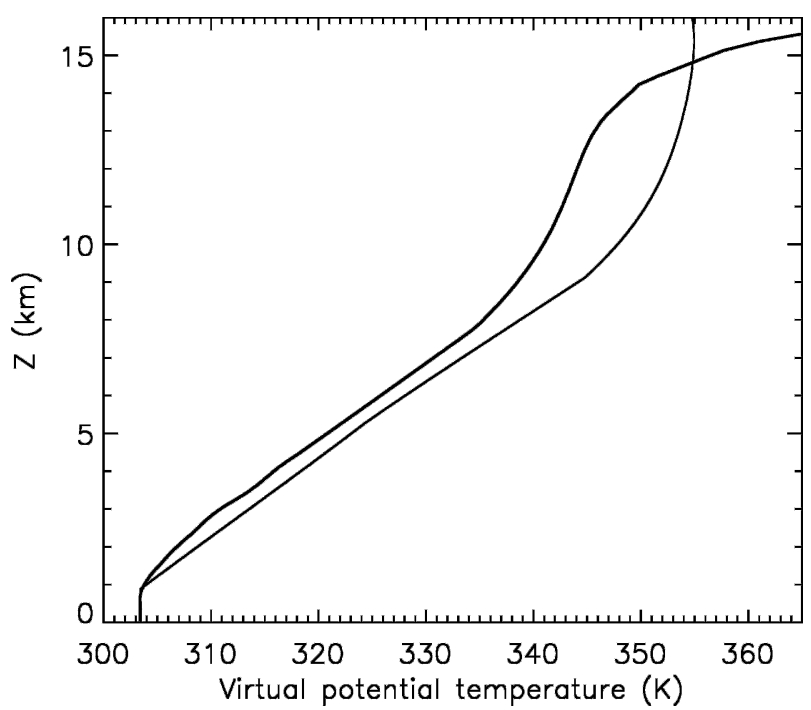

FIG. 2. Domain-mean profiles for a 30-min period during day 2 of environmental virtual potential temperature (thick) and the density potential temperature of a cloudy cloud-base particle lifted adiabatically without mixing (all condensates are retained in the parcel).

and cloud fraction is provided in Fig. 1b-d. During the first $12 \mathrm{~h}$, a nearly statistically steady shallow convective regime is established. As the surface fluxes increase, convection deepens. The inversion originally located at $2 \mathrm{~km}$ gets eroded and increases in height. At the end of day 2, the inversion is no longer discernable. Convection develops into a cumulus congestus regime. At this time there is substantial convectively available potential energy (CAPE) for an undilute near-surface air parcel to rise to the upper troposphere, as shown in Fig. 2, but deep convection does not occur because the midtroposphere is still relatively dry. A dry midtroposphere is unfavorable to deep convection because lateral entrainment of drier environmental air by the rising plumes leads to more evaporative cooling, and hence negative buoyancy (Derbyshire et al. 2004). Deep convection develops after the middle troposphere is moistened by the cumulus congestus, as indicated in Fig. 1 by the appearance of substantial upper tropospheric anvil clouds around the beginning of day 5. Figure 1 clearly shows that the thermodynamic structure evolves continuously during the transition, without a discrete eruption into deep convection, providing justification for developing unified shallow and deep cumulus parameterizations.

In the following discussion, we shall refer to the time period from 9 to $12 \mathrm{~h}$ as the shallow cumulus regime, day 2.5 to day 3.5 as the cumulus congestus regime, and the last day as the deep cumulus regime. Instantaneous three-dimensional (3D) fields were saved after every 20 min of simulated time, and horizontally averaged quantities were sampled every $30 \mathrm{~s}$ and saved as 30 -min averages.

To further examine the effect of midtroposphere moisture, we have conducted an additional experiment, where the simulation was restarted at the end of day 2.5 of the control experiment. Horizontally uniform values were added to the $q_{v}$ field above $3 \mathrm{~km}$ so that the domain averaged $q_{v}$ profile above $3 \mathrm{~km}$ matches that at the end of day 5 of the nominal run (Fig. 3a). In this simulation, deep convection develops rapidly (Fig. 3b), confirming the role of midtroposphere dryness in delaying the development of deep convection in the control case (Fig. 1d).
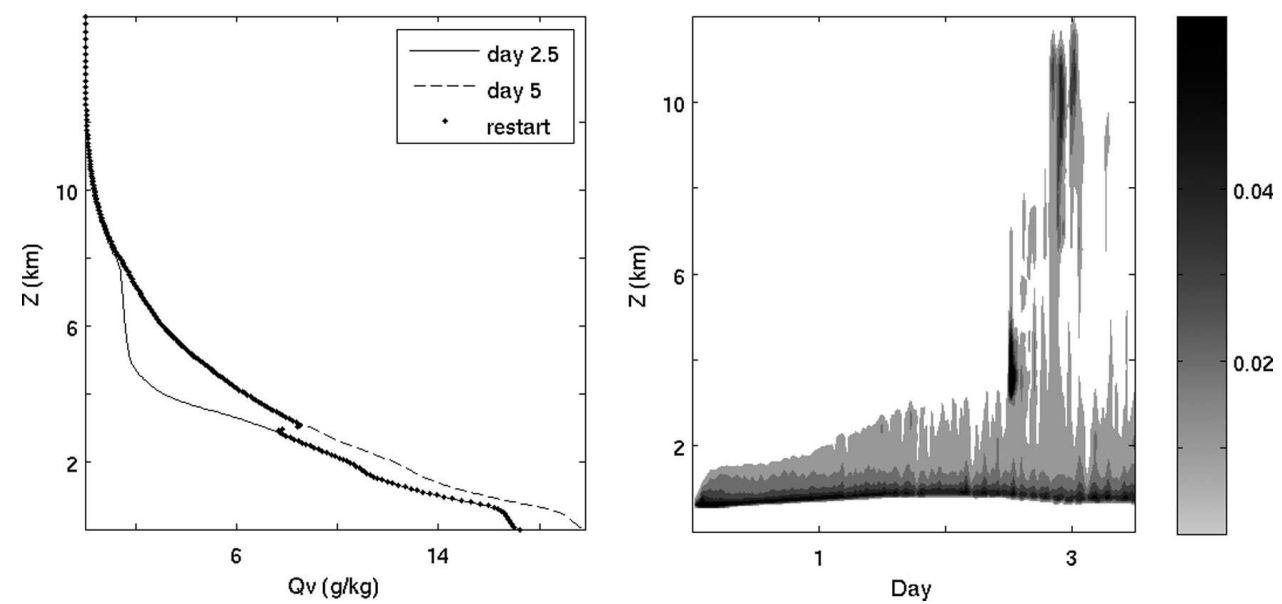

FIG. 3. (a) Domain-averaged $q_{v}$ profile at the end of day 2.5 (solid) and day 5 (dashed) of the control run. The difference between the dotted line and the solid line was added to the new run, which restarted at the end of day 2.5 of the nominal run. (b) The evolution of cloud fraction profile in the restarted run. The first 2.5 days are the same as the nominal run shown in Fig. 1d. 
While one could choose to simulate the transition from shallow to deep cumulus convection in the more realistic setting of the continental diurnal cycle (Grabowski et al. 2006), the present idealized experimental setup was chosen so that the transition occurs slowly (over 2-3 days instead of 1-2 h). This affords us adequate sampling with a relatively small domain. Equivalent sampling in a diurnal cycle study requires a domain that is too large for the memory of our present computer cluster to accommodate.

\section{Analyses and results}

In our analysis we will extensively use thermodynamic variables that are (within the numerical formulation of the simulation) conserved in adiabatic fluid motions including phase changes of water, and which are also approximately linearly mixing. Two such variables introduced in section 2 were SAM's prognostic thermodynamic variables, the total water specific humidity $q_{t}$ and the liquid-ice static energy $s_{l i}$. We shall exclude the effect of precipitating water from $s_{l i}$; that is, redefine $s_{l i}=c_{p} T+g z-L q_{n}-L_{f} q_{i}$, so that it is better conserved in the presence of precipitation. It is useful in addition to define two further variables. The first is the frozen moist-static energy $h=s_{l i}+L q_{t}=c_{p} T+g z+$ $L q_{v}-L_{f} q_{i}$, hereafter referred to as MSE. This variable is conserved by the model even for air parcels in which there is production or evaporation of liquid precipitation. The second is the liquid water virtual potential temperature $\theta_{v l}=\theta_{l}\left(1+0.61 q_{v}\right)$, where $\theta_{l}=(T-$ $\left.L q_{l} / c_{p}\right)(1000 \mathrm{hPa} / p)^{R / c p}$, and $R$ is the specific gas constant. In the numerical model, this variable is approximately but not exactly conserved and linearly mixing. It is useful for comparing the densities of air parcels from different levels, after they are adiabatically brought to a common level at which they are unsaturated.

\section{a. Cloud-base mass flux}

An important component of a mass-flux scheme is to determine the cloud-base mass flux. While it has been popular to build the closure assumption solely on CAPE (Arakawa and Schubert 1974; Bechtold et al. 2001; Fritsch and Chappell 1980), it is clear that during the transition from shallow to deep cumulus, such a formulation is not valid. Figure 4 shows the time evolution of 1) the cloud-base mass flux and 2) the CAPE. CAPE is computed by reversibly displacing an air parcel with the mean thermodynamic properties of cloudy cloud-base air parcels. At $\sim 28 \mathrm{~h}$, the displaced undiluted parcel overcomes the trade inversion and gains
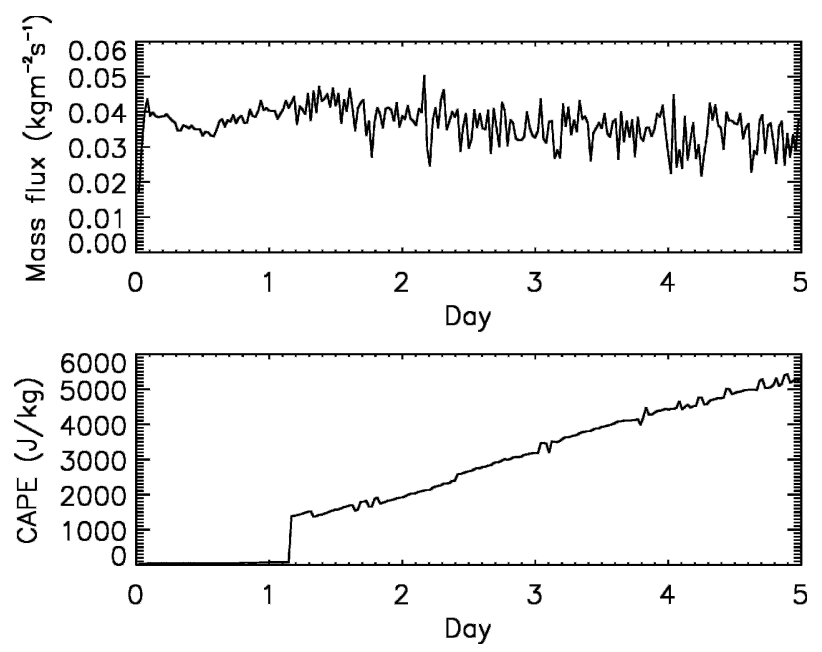

FIG. 4. Time evolution of (a) the cloud-base mass flux and (b) CAPE. CAPE is computed by reversibly displacing an air parcel with the mean thermodynamic properties of cloudy cloud-base air parcels.

access to the conditional instability above the inversion, leading to the sudden increase in CAPE. After that, CAPE continues to increase and gains another factor of 3 by the end of the simulation. In contrast, the cloudbase mass flux is slightly smaller during the latter periods of the simulation. The cloud base here is identified as the level of maximum cloud fraction. Variations in the cloud-base mass flux were also found to be small in a study of the transition of shallow to deep convection transition over land (Grabowski et al. 2006). A recent CRM study of the diurnal cycle of shallow cumulus convection (Neggers et al. 2004) also reached negative conclusions about the CAPE closure.

A more recent closure suggestion is that the cloudbase mass flux is regulated through its interaction with the weak stable layer atop the subcloud layer. This weak stable layer provides convective inhibition (CIN) to subcloud layer air parcels. Borrowing a concept from statistical mechanics, Mapes (2000) considered the role of subcloud layer fluctuations in overcoming CIN and triggering convection, and proposed that the cloud base mass flux be parameterized in the form $W \exp (-k \mathrm{CIN} /$ $W^{2}$ ), where $W$ is some measure of typical updraft vertical velocity at cloud base and $k$ is a constant. When CIN is too small, cloud mass flux increases and causes stronger compensating subsidence, which heats the cumulus layer and increases CIN until a balance is established. Bretherton et al. (2004) demonstrated the viability of this approach as a combined mass-flux closure and trigger implemented in a parameterization of shallow cumulus convection, taking $W^{2}$ equal to the sub- 
cloud layer turbulent kinetic energy (TKE) prognosed by a turbulence parameterization. We now use our CRM simulation to support its plausibility over the entire shallow to deep convection transition.

Figure 5 shows the buoyancy profiles for a reversibly displaced parcel with the averaged thermodynamic properties of cloud base cloudy air parcels. The results are averaged over the shallow (solid), congestus (dotted), and deep cumulus (dashed) periods, based on 30min average soundings. CIN can be estimated from the area of negative buoyancy above the originating level. We see that CIN is smaller in the shallow cumulus regime compared to the other two regimes. In Fig. 6, CIN so estimated is shown as a function of time. It increases substantially from initial values of $\sim 0.12 \mathrm{~J} \mathrm{~kg}^{-1}$ in the shallow convection regime to about $0.5 \mathrm{~J} \mathrm{~kg}^{-1}$ in the deep convection regime. We have also plotted the TKE estimated at the $475-\mathrm{m}$ level, just below the area of negative buoyancy for the displaced parcel. It also shows higher values for the congestus and deep cumulus regimes, presumably due to the effect of cold-pool circulations driven by convective downdrafts. The CIN to TKE ratio is fairly constant in comparison. Since the cloud-mass flux is also fairly constant, this is consistent with the $\mathrm{TKE}^{1 / 2} \exp (-\mathrm{CIN} / \mathrm{TKE})$ formulation (shown as dashed), though a full CRM test of that formulation would need to span a greater range of variations in the CIN to TKE ratio.

\section{b. Cloud-base properties}

Another important component of a mass-flux scheme is the cumulus cloud-base properties, because they pro-

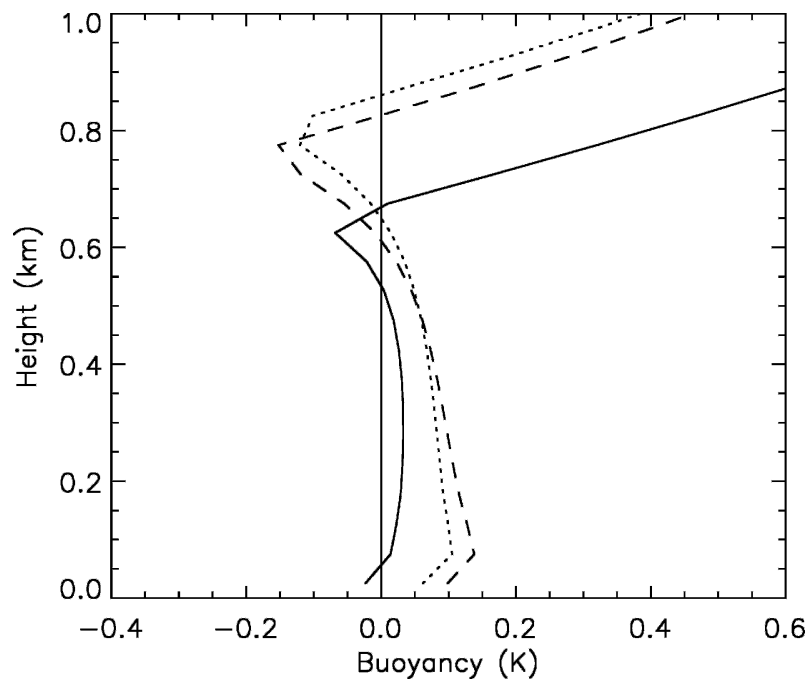

FIG. 5. The buoyancy profiles produced by reversibly displacing an air parcel with the mean thermodynamic properties of cloudy cloud-base air parcels for the shallow (solid), congestus (dotted), and deep (dashed) cumulus periods.

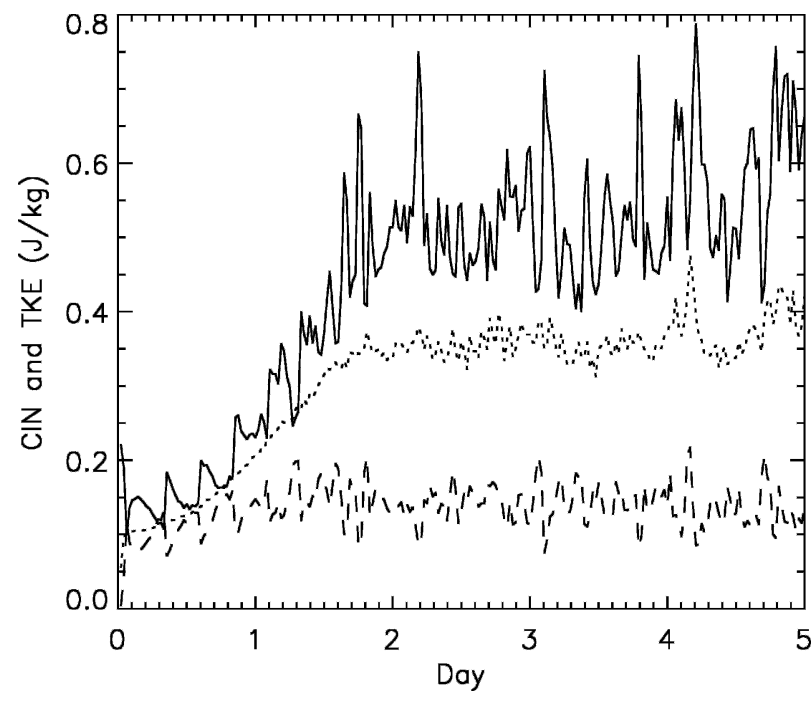

FIG. 6. Time evolution of CIN (solid) and the turbulent kinetic energy (TKE) at the 475-m level (dotted). The dashed line shows $\mathrm{TKE}^{1 / 2} \exp (-\mathrm{CIN} / \mathrm{TKE})$, plotted on the same scale but with the unit of $\mathrm{m} \mathrm{s}^{-1}$.

vide the starting conditions for cumulus mixing processes in the layer above. In Fig. 7, we compare the joint probability distribution function (PDF) of $q_{t}$ and $\theta_{v l}$ for air parcels in the lowest model level above the surface, in a noncloudy layer just below the cloud base, and at the cloud base, conditionally sampled for cloudy and noncloudy grid points. These are computed from all the

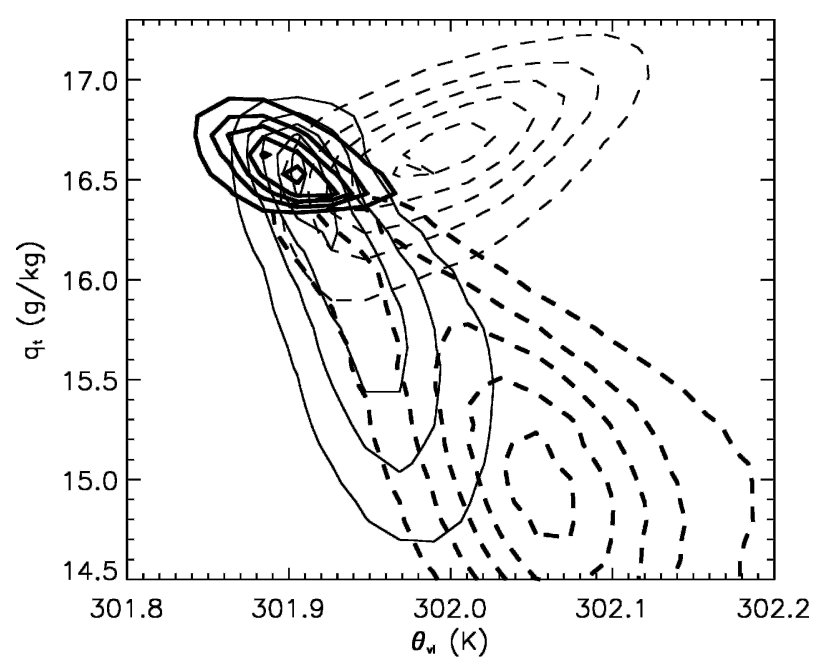

FIG. 7. Joint PDF of total water $q_{t}$ and liquid water virtual potential temperature $\theta_{v l}$ at the surface (thin, dashed), in the noncloudy layer just below the cloud base (thin, solid), and at the cloud base, conditionally sampled for cloudy (thick, solid) and noncloudy (thick, dashed) grid points. The PDFs are normalized by their maximum values and are plotted with contour values of $0.9,0.7,0.5,0.3$, and 0.1 . 


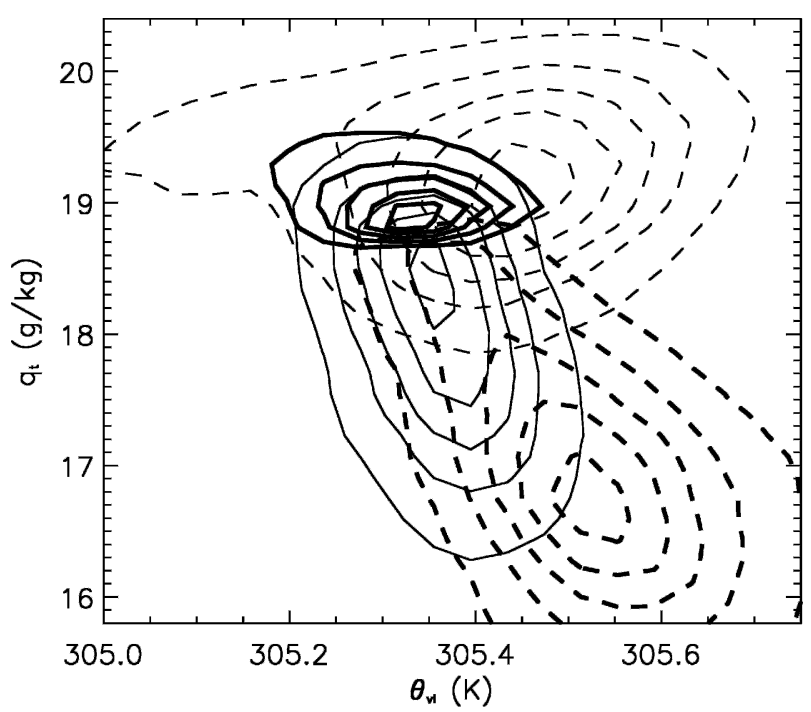

FIG. 8. The same as Fig. 7, except for a 3-h period in the deep cumulus regime.

instantaneous 3D fields saved during the shallow cumulus regime. The cloud base is chosen to be the level of maximum cloud fraction in the lower troposphere, and is separated from the noncloudy layer just below it by $100-150 \mathrm{~m}$. Figure 7 indicates that for shallow convection, cloudy parcels at the cloud base are simply the moistest subcloud layer parcels and tend to have $q_{t}$ similar to the parcels near the surface. The cloudy parcels tend to be slightly negatively buoyant before they rise up through the cloud base (cf. thick and thin solid contours in Fig. 7). This is consistent with the negative buoyancy fluxes found just below cloud base documented in earlier CRM simulations (e.g., Siebesma et al. 2003). These are associated with entrainment by non-cloud-forming subcloud-layer eddies of higher- $\theta_{v l}$ air from above the weak transition-layer inversion that forms at the cloud base. The positive slope in the surface air properties of $\sim 0.2 \mathrm{~K}\left(\mathrm{~g} \mathrm{~kg}^{-1}\right)^{-1}$ is consistent with the ratio between the surface fluxes of $\theta_{v l}$ and $q_{t}$. Although we did not use sophisticated back-trajectory calculations, the cloudy parcels at the cloud base have substantial positive buoyancies compared to their surroundings and can be safely assumed as the starting points for the plumes. These results for shallow convection are largely consistent with previous modeling studies (e.g., Lin 1999) and aircraft observations (Pennell and Lemone 1974).

The results for the deep cumulus regime are shown in Fig. 8, and are very similar to those of the shallow cumulus regime, except in the surface layer, where we now see the signature of cold pools with substantial negative buoyancy. Results for the congestus regime lie between the two (not shown). One remarkable feature common to all cases is the tightness of the PDF of the thermodynamic properties of the cloudy air at the cloud base. The spreads in $\theta_{v l}$ and $q_{t}$ (defined by where the probability drops to half of its maximum value) for the shallow cumulus regime are about $0.03 \mathrm{~K}$ and $0.1 \mathrm{~g}$ $\mathrm{kg}^{-1}$, respectively, while those for the deep cumulus regime are about $0.1 \mathrm{~K}$ and $0.2 \mathrm{~g} \mathrm{~kg}^{-1}$. This suggests that using a single set of cloud-base thermodynamic properties for all plumes in a cumulus scheme is a reasonable simplification if a plume model (next section) with a constant entrainment rate for each plume is used. If potential feedbacks such as that between entrainment rate and vertical velocity were included, variability due to the narrow distributions of thermodynamic properties at the cloud base would become more important (Neggers et al. 2002).

\section{c. Comparison of CRM in-cloud PDFs to a spectral entraining plume model}

A central component of a mass-flux convective parameterization is a cloud model within that predicts how mass flux and properties of convective updrafts (such as condensate amount, buoyancy, and vertical velocity) evolve with height. Commonly this is formulated in terms of a bulk or spectral entraining-detraining plume model. In such a model, the vertical profiles of upward cumulus mass flux $M_{u}=\rho \sigma w$ and a typical updraft or plume property $\psi_{u}$ are described by

$$
\frac{\partial M_{u}}{\partial z}=M_{u}(\varepsilon-\delta)
$$

and

$$
\frac{\partial \psi_{u}}{\partial z}=\varepsilon\left(\bar{\psi}-\psi_{u}\right)+S_{\psi},
$$

where $\rho$ is the updraft air density, $\sigma$ is the fractional area occupied by the updrafts, $w$ is the updraft velocity, $\varepsilon$ and $\delta$ are the fractional entrainment and detrainment rates, the overbar denotes a horizontal average, and $S_{\psi}$ is the source term for $\psi$, including effects of phasechange, precipitation, etc. A bulk plume model uses one such plume, and a spectral plume model partitions the cloud-base mass flux between multiple plumes each following (1)-(3), but with different $\varepsilon$ and $\delta$. Diverse approaches have been proposed for specifying $\varepsilon, \delta$, and their relation in bulk plume models, using empirical relations, scaling, and buoyancy-sorting arguments.

As discussed by Siebesma et al. (2003), many cloud models also make use of a plume vertical velocity equation of the form 


$$
\frac{1}{2} \frac{\partial}{\partial z} w_{u}^{2}=a B-b \varepsilon w_{u}^{2}
$$

where $B$ is the updraft buoyancy, $a$ is a virtual mass coefficient, and $b$ is a drag coefficient. For instance, Bretherton et al. (2004) used $a=1$ and $b=2$. This vertical velocity equation enters into calculations of microphysics and of penetrative entrainment associated with plumes overshooting their level of neutral buoyancy.

As mentioned in the introduction, past CRM studies have examined entrainment and detrainment processes in individual shallow and deep cumuli by combining analyses of parcel trajectories and conserved variables. These approaches, together with an extensive body of aircraft observations have yielded insights into when, where, and how entrainment is occurring in evolving cumuli. However, because they require identification and tracking of individual cloud parcels, they are cumbersome. For this study, we have devised a simpler analysis technique to compare the PDF of cloud properties in our CRM simulation to a spectral plume model. At each height, we conditionally sample the cloudy air parcels, defined as grid points with total cloud water/ice specific humidity $q_{n}>0.01 \mathrm{~g} \mathrm{~kg}^{-1}$. These grid points are then binned according to their frozen MSE $h$ as defined at the beginning of this section. MSE was chosen for this purpose because it is conserved in adiabatic fluid motions even when rain is being produced or evaporated. (Ice-phase precipitation is a small MSE source term but is not important at temperatures above freezing, where most of our analysis occurs.) Thus an in-cloud MSE different from the cloud-base MSE is a reliable indicator of turbulent mixing between cloud and environmental air even for precipitating convection. The total mass flux and composites of quantities such as buoyancy and vertical velocity are computed for each bin with more than 100 data points, and plotted as a function of MSE and height.

Figure 9 shows the resulting MSE binning of the cloud mass flux at each height for the shallow cumulus regime. At the mean cloud base of $\sim 0.7 \mathrm{~km}$, almost all the cloud mass flux is carried in air parcels with $h / c_{p}$ lying in a very narrow range of $\pm 0.5 \mathrm{~K}$ (as discussed in the previous section) around a mean value $h_{B} / c_{p}=343$ $\mathrm{K}$. However, even as little as $200 \mathrm{~m}$ above cloud base, the cloudy updrafts have systematically lower MSE, and air with the undilute cloud-base MSE contributes negligibly to the mass flux. The typical cloudy updraft MSE continues to decrease steadily with height. This emphasizes the critical role of dilution with environmental air in setting the PDF of cloud properties. Near

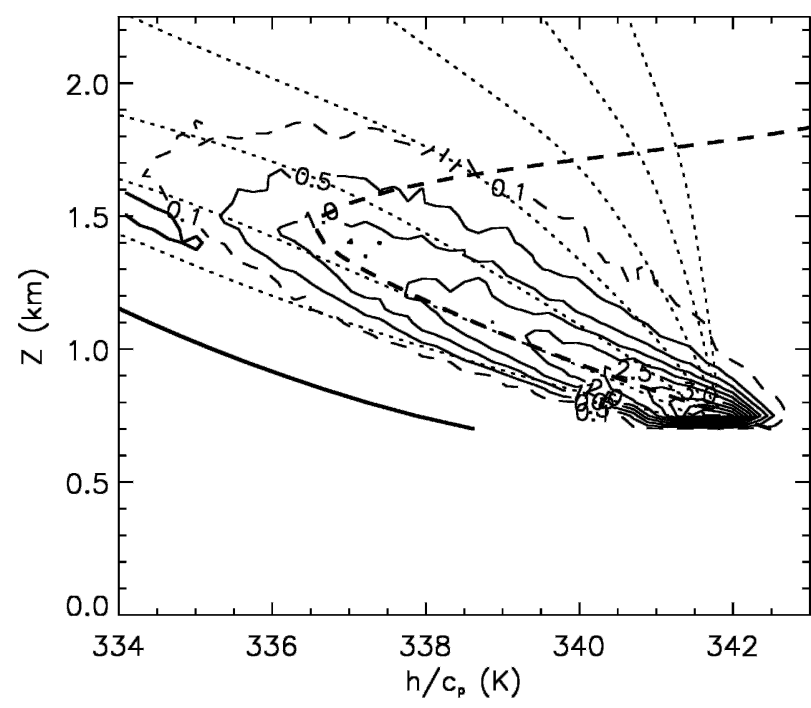

Fig. 9. The cloudy updraft/downdraft mass flux at each height binned by their MSE $h$ (expressed as $h / c_{p}$ in temperature units). The bin size is $0.18 \mathrm{~K}$. The interval of the solid contours is $0.5 \mathrm{~g}$ $\mathrm{m}^{-2} \mathrm{~s}^{-1} \mathrm{bin}^{-1}$, the dashed contour is for $0.1 \mathrm{~g} \mathrm{~m}^{-2} \mathrm{~s}^{-1} \mathrm{bin}^{-1}$, and the thick solid contour is for $-0.1 \mathrm{~g} \mathrm{~m}^{-2} \mathrm{~s}^{-1} \mathrm{bin}^{-1}$, representing the downdrafts. The dotted lines are the MSEs predicted by entraining plume models with fractional entraining rates of (from right to left) $0.0625 \mathrm{~km}^{-1}, 0.125 \mathrm{~km}^{-1}, 0.25 \mathrm{~km}^{-1}, \ldots, 4 \mathrm{~km}^{-1}$. The thick solid line to the far left is the domain mean MSE profile, and the thick dashed line is the domain mean saturation MSE profile.

the inversion, air with low MSE contributes to downward mass fluxes (thick contours).

It is illuminating to interpret Fig. 9 in terms of an entraining plume model. Using the plume dilution Eq. (2) with $\psi=h$, diluting it with the domain-average $h$ profile (solid curve), assuming no source term (fully justifiable only below the freezing level), and integrating upward from a cloud base MSE of $h_{B}$, we can predict updraft $h_{u}(z ; \varepsilon)$ as a function of entrainment rate $\varepsilon$. The dashed lines in Fig. 9 are the predicted MSE for entraining plumes with different $\varepsilon$. Equivalently, we can present this model data by calculating the effective entrainment rate $\varepsilon$ corresponding to each $h_{u}$ at each height, and binning the cloudy updraft grid points in terms of $\varepsilon$ instead of MSE. This provides a view of the cloudy updrafts as an ensemble of entraining "plumes" 2 with a range of entrainment rates. Results from the CRM can thus be directly compared with predictions of an entraining plume model. As the atmosphere slowly evolves with time in our experiment, the mapping between entrainment rate and MSE is done for each 3D

\footnotetext{
${ }^{2}$ For clarity, the word plume will be put in quotation marks when it is used to interpret the CRM results (i.e. "plume").
} 
snapshot. The results are then averaged over the three regimes. Because our goal is to compare simple plume models against the CRM simulation, we have neglected the small but finite spread in the thermodynamic properties of cloudy cloud-base air and used a single set of properties to initiate the plumes. This spreads the mass flux into a wider range of $\varepsilon$, especially near the cloud base; that is, we are effectively using a wider spectrum of plumes to account for the variations in the initial thermodynamic properties. The initial vertical velocity is set to zero when calculating the vertical velocity at higher levels in all plumes. As in Lin and Arakawa (1997), we adopt the view that the plume spectrum should be thought of as a representation of the ensemble mixing dynamics of subcloud elements, rather than each plume corresponding to one cumulus cloud. However, the current procedure, unlike Lin and Arakawa (1997), can provide an estimate of the mass flux and mean properties for "plumes" within a given range of effective entrainment rates without the complication of extensive trajectory calculations.

The resulting $\varepsilon$-binned profiles of "plume" mass flux, buoyancy, and vertical velocity are shown in Figs. $10 \mathrm{a}, \mathrm{c}, \mathrm{e}$. Note that $\varepsilon$ is binned and plotted using a logarithmic scale. Figure 10a restates the results of Fig. 9. Most of the mass flux is associated with $\varepsilon$ values of 1-3 $\mathrm{km}^{-1}$, consistent with previous studies (e.g., Siebesma et al. 2003). The dashed line in Fig. 10a indicates that little mass flux is carried up by "plumes" with effective entrainment rates less than $\sim 0.3 \mathrm{~km}^{-1}$. Figure $10 \mathrm{c}$ shows that the overall buoyancy of the "plumes" is remarkably small; for the "plumes" that carry most of the mass flux, it is less than $0.5 \mathrm{~K}$. "Plumes" with smaller entraining rates have greater buoyancies $(0.5-1 \mathrm{~K})$, and can achieve higher vertical velocities. The more heavily entraining "plumes" tend to become negatively buoyant at lower altitudes. Above the zero buoyancy level of a "plume" (indicated by the white curve in Fig. 10c), the mass flux decreases rather rapidly - a manifestation of buoyancy sorting. The "plume" vertical velocity also starts to decrease, though not so dramatically. These results are largely consistent with our existing knowledge of shallow cumulus convection (Siebesma et al. 2003), except now expressed in a plume ensemble view.

As an illustrative example, we compare these results with the spectral entraining plume model of (Arakawa and Schubert 1974), in which for any given $\varepsilon$, the detrainment rate $\delta$ is assumed to be zero when the plume is positively buoyant, but becomes infinite when the plume becomes negatively buoyant. For such a plume, (1) implies the mass flux increases exponentially with height proportional to $\exp (\varepsilon z)$, then sharply cuts off at its level of neutral buoyancy. The updraft MSE is computed using (2) exactly as above, starting from the CRM-derived cloud base $h_{B}$, and assuming no source/ sink terms except dilution with mean environmental air and conversion of cloud ice to precipitating ice (see below). The updraft $q_{t}$ is computed similarly. Once $h$ and $q_{t}$ are known at a given height from (2), the condensate specific humidity, the density temperature, and hence the buoyancy of the parcel can be computed. For the precipitating (congestus and deep convection) regimes, we assume that net loss of condensate associated with the precipitation processes has an efficiency of $0.15 \mathrm{~km}^{-1}$. This factor includes both the loss of condensate by precipitation from a grid box and the gain of condensate as precipitation falls from grid boxes above. It is empirically determined for this experiment and is expected to vary when, for example, vertical shear is included. A more sophisticated scheme would be appropriate for an operational cumulus parameterization, but this simple approach suffices for illustration. The loss of $q_{n}$ provides a sink term for $q_{t}$ and the loss of $q_{i}$ provides a small source term for $h$. The vertical velocity of a plume with a specified $\varepsilon$ is then obtained by integrating (3) upward from the cloud base using $a=1$, $b=2$ as in Bretherton et al. (2004). In addition, the mass flux for each $\varepsilon$ is chosen to match the CRM simulation $200 \mathrm{~m}$ above the cloud base. Discretization of the equations follows appendix A of Bretherton et al. (2004).

The predicted mass flux, buoyancy, and vertical velocity for the shallow cumulus case from this entraining plume model are shown in Figs. 10b,d,f. The predicted buoyancy (Fig. 10d) is in very good agreement with those from the CRM. This is expected, since by construction the CRM will have the same $h$ for a given $\varepsilon$-bin as the entraining plume, the updraft $q_{t}$ in these nonprecipitating cumuli should behave similarly to $h$, and buoyancy is determined by $h$ and $q_{t}$. Predicted vertical velocity (Fig. 10f) is also in good agreement with those from the CRM, though it tends to weaken slightly more rapidly with increasing $\varepsilon$. The simple formulation (3) thus appears adequate in predicting the updraft vertical velocity. However, Fig. 10b shows that the entraining plume model overpredicts the mass flux as the plume approaches the zero buoyancy level; while the mass flux of a "plume" from the CRM simulation starts to decrease as it approaches the zero buoyancy level, the mass flux of an entraining plume continues to increase exponentially until its buoyancy becomes negative. A similar conclusion was reached previously by Zhao and Austin (2005) in a study of individual shallow cumuli. 

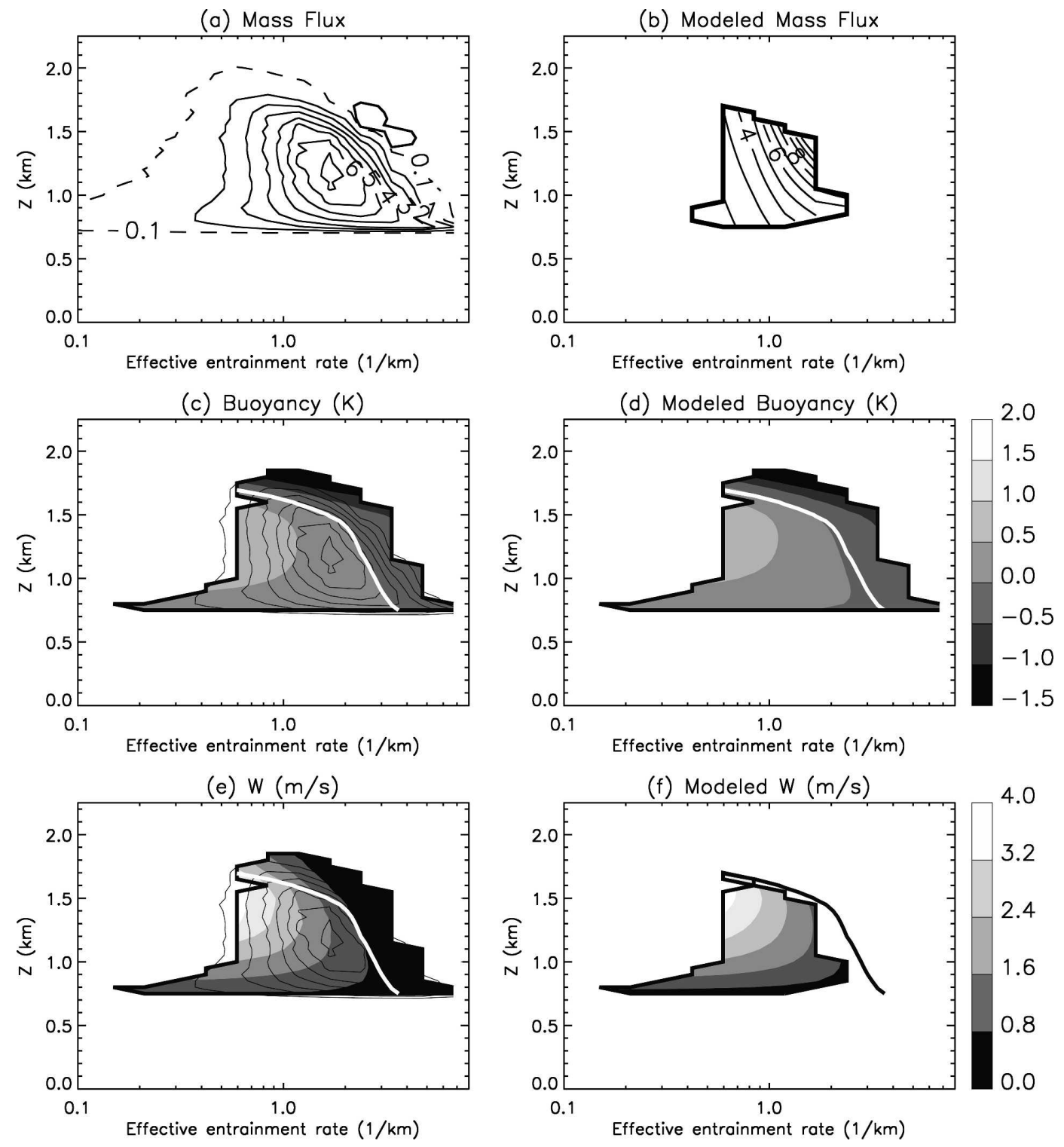

FIG. 10. (a) The cloudy updraft/downdraft mass flux at each height binned by their effective entrainment rates. The bin boundaries have entrainments rates of $2^{(i / 2)-4}$, for $i=0,1,2, \ldots, 16$. The interval of the solid contours is $1 \mathrm{~g} \mathrm{~m}^{-2} \mathrm{~s}^{-1} \mathrm{bin}^{-1}$, while the $0.1 \mathrm{~g} \mathrm{~m}^{-2} \mathrm{~s}^{-1} \mathrm{bin}^{-1}$ contour is dashed, and the thick solid contour is for $-0.1 \mathrm{~g} \mathrm{~m}^{-2}$ $\mathrm{s}^{-1}$ bin $^{-1}$. (b) Same as (a), except for the predicted mass flux of entraining plumes from the cloud base up to the level where the predicted buoyancy becomes negative. We have scaled the modeled mass flux of each plume so that it matches that from the CRM simulation $200 \mathrm{~m}$ above the cloud base. To compare with (a), we have only plotted regions where values in (a) exceed $1 \mathrm{~g} \mathrm{~m}^{-2} \mathrm{~s}^{-1} \mathrm{bin}^{-1}$. (c) Composite buoyancy for each bin from the CRM simulation. The mass-flux contours are overplotted. (d) Same as (c), except for the modeled buoyancy and without the mass-flux contours. (e) Composite vertical velocity for each bin from the CRM simulation. (f) Same as (e), except for the modeled vertical velocity up to the level where the modeled buoyancy becomes negative. In (c) and (e), composite values are assigned only to bins that contain more than 100 data points. The zero buoyancy line estimated from the CRM output is the thick white curve in (c), (d), (e), and the thick black curve in (f).

Corresponding results for the deep cumulus regime are shown in Figs. 11 and 12. Results for the condensate amount are also included (Figs. 12g,h). In this case, the plumes extend into the region where the domain-mean MSE increases with height (above $\sim 5.5 \mathrm{~km}$ as seen in the thick, solid line in Fig. 11). When the plume MSE is lower than the domain-mean MSE, it is possible for plumes of different entrainment rates to arrive at the same height with the same MSE, so that one can no longer unambiguously assign an effective entrainment 


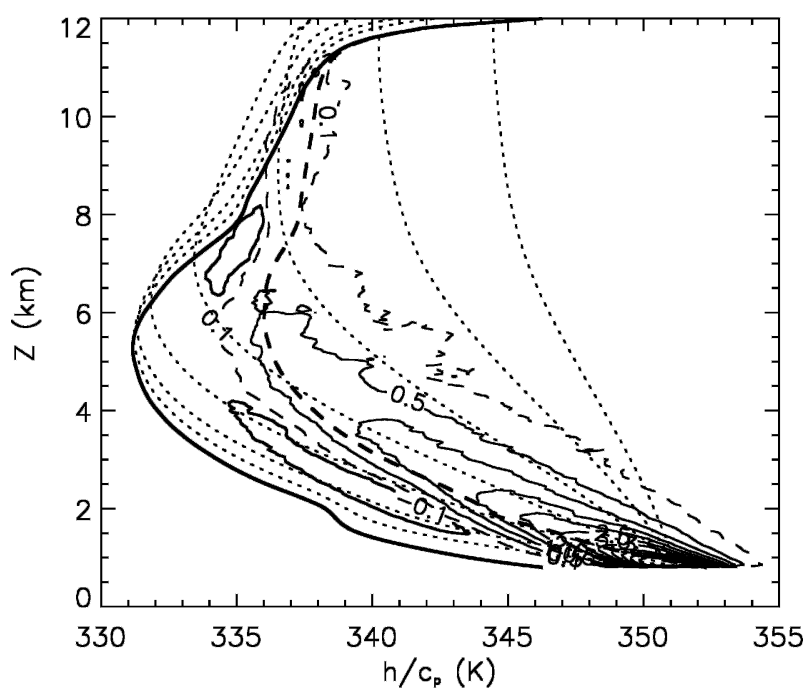

FIG. 11. Same as Fig. 9, except for the deep cumulus regime and for a bin size of $0.5 \mathrm{~K}$ in $h / c_{p}$.

rate to a grid point. Therefore, we shall only examine the results below $8 \mathrm{~km}$, where most of the cloudy updrafts have MSE values greater than the domain mean.

The salient features for the deep cumulus regime are rather similar to those of the shallow cumulus regime, with a few notable differences. While in shallow convection, the majority of the mass flux is carried up by "plumes" with entrainment rates around $2 \mathrm{~km}^{-1}$, over the same range of heights (between the cloud base and $\sim 2 \mathrm{~km}$ ) in the deep cumulus regime, the "plumes" have smaller entrainment rates centered around $1-1.5 \mathrm{~km}^{-1}$ (Fig. 12).

Despite a general tendency for smaller entrainment rates in the deep cumulus regime, there is still little mass flux carried up by "plumes" with effective entrainment rates less than $\sim 0.1-0.2 \mathrm{~km}^{-1}$ (see the dashed line in Fig. 12a). Therefore, undiluted air parcels do not play a significant role in the simulated convection, contrary to the assumption made in many deep cumulus schemes (Emanuel 1991; Zhang and McFarlane 1995). As in the shallow convection case, the overall buoyancy of the "plumes" that carry most of the mass flux is small, less than $1 \mathrm{~K}$, although the least entraining "plumes" can attain buoyancies around $2 \mathrm{~K}$ and vertical velocities greater than $10 \mathrm{~m} \mathrm{~s}^{-1}$ in the midtroposphere. As the more heavily entraining "plumes" terminate at lower altitudes, the "plume" population shifts toward lower entrainment rates.

The buoyancy and vertical velocity predicted by the entraining plume model appears to be in good agreement with the CRM simulation for the deep cumulus regime as well (Figs. 12d,f), although the model overpredicts to some extent the buoyancy and vertical ve- locity at higher altitude for plumes with small entrainment rates. Our simple parameterization of the condensate removal (a constant removal rate of $0.15 / \mathrm{km}$ ) is broadly successful, though it underestimates the condensate loading at low altitudes while overestimating it at higher altitudes. As in the shallow cumulus regime, the entraining plume model overpredicts the mass flux as the plume approaches the zero buoyancy level. The mass flux of a "plume" from the CRM simulation starts to decrease as it approaches the zero buoyancy level, while the mass flux of an entraining plume continues to increase exponentially.

It thus appears that a model with a spectrum of entraining plumes can in fact reproduce most features of the cloudy updrafts simulated by the CRM in both the shallow and deep convection regimes, except the decrease in mass flux as the plumes approach their levels of zero buoyancy. A potential remedy is to replace the spectrum of entraining plumes by a spectrum of entraining-detraining plumes in which detrainment gradually starts to occur as soon as the plume buoyancy starts to decrease with height, a phenomenon noted by Bretherton and Smolarkiewicz (1989) and others. This would not affect the results for buoyancy and vertical velocity but would reduce the mass flux through increased detrainment as the plume loses its buoyancy.

A treatment of the downdrafts, necessary for precipitating convection, is not addressed here. In fact, the present simulation never has very intensive precipitation (Fig. 1b), and downdrafts are of modest strength (Figs. 11 and 12). While the similarity between shallow convection and the deep convection simulated here viewed as an ensemble of updraft plumes is encouraging, further extension to deep convection with stronger precipitation and downdrafts is needed in order to develop a unified plume model for both shallow and deep convection.

The overall smallness of the buoyancy (Fig. 12c) is consistent with observational studies (e.g., Igau et al. 1999). We suggest that this may be maintained by the following feedback, both in shallow and deep convection. Suppose that at a certain time, the buoyancy of the bulk of the updrafts is substantially positive. These updrafts will then achieve larger vertical velocities and overshoot their levels of neutral buoyancy more strongly. This has the effect of entraining more of the overlying warmer air downward, which warms the environment and reduces the buoyancy of the updrafts.

\section{d. Partitioning of cloud-base mass flux between $\varepsilon$ bins}

If the cloud ensemble is represented as a spectrum of plume types of different entrainment rates $\varepsilon$, a key 
(a) Mass Flux

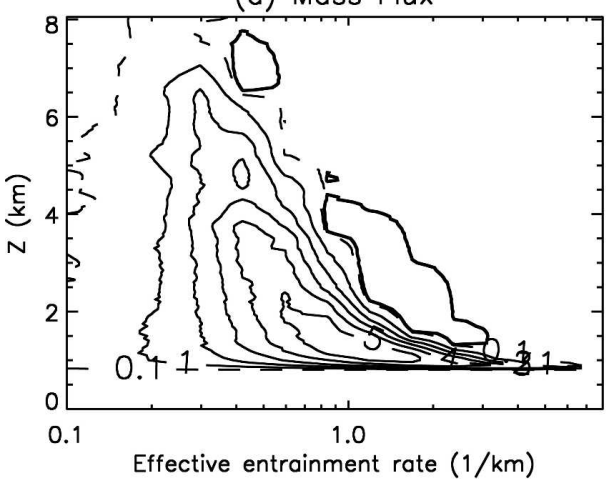

(c) Buoyancy (K)

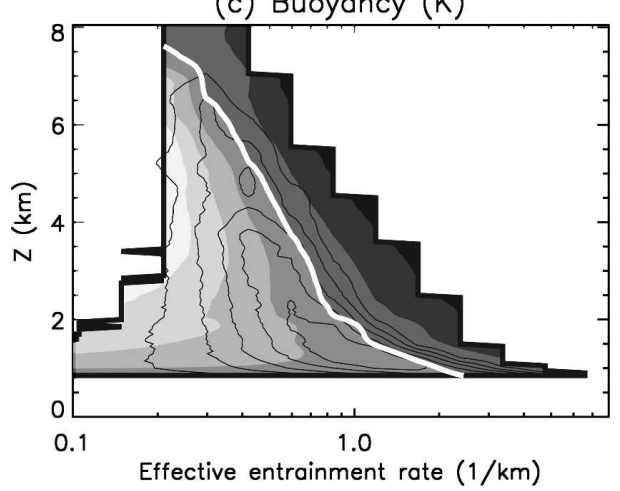

(e) $W(\mathrm{~m} / \mathrm{s})$

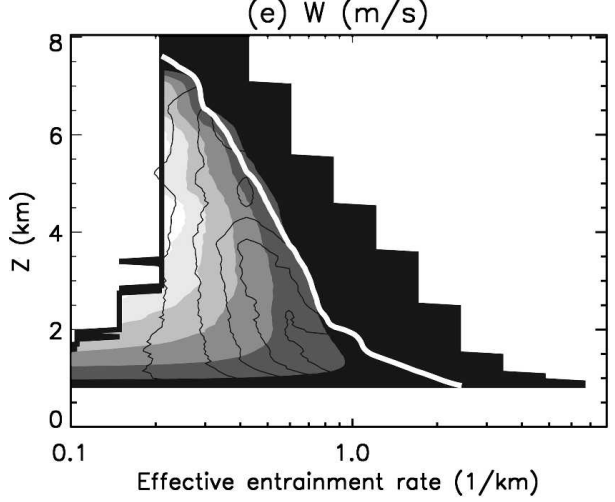

(g) condensate $(\mathrm{g} / \mathrm{kg})$

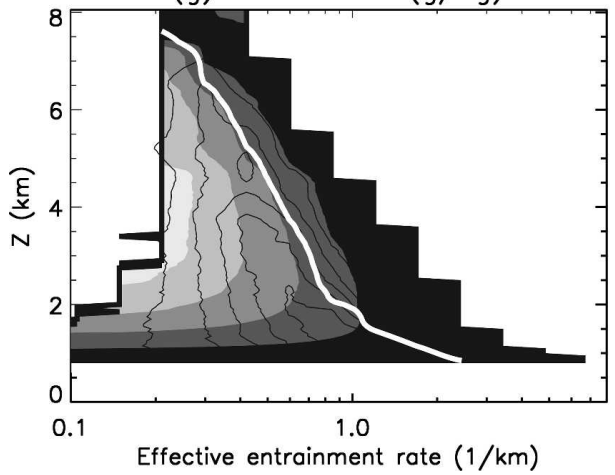

(b) Modeled Mass Flux

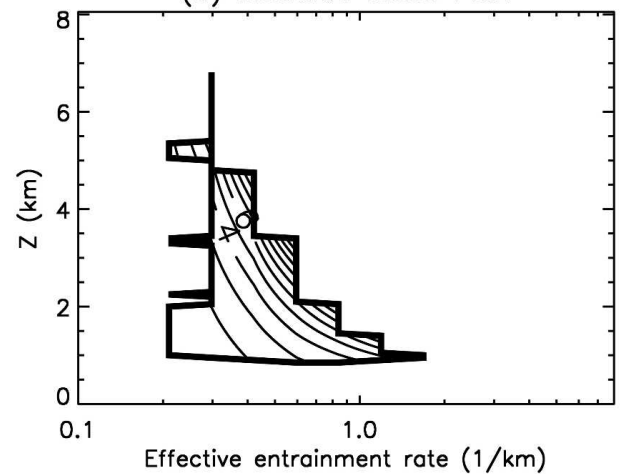

(d) Modeled Buoyancy (K)

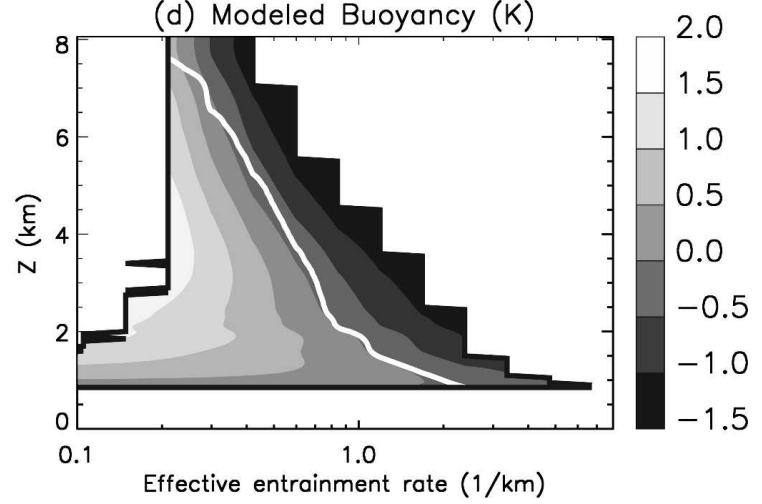

(f) Modeled W (m/s)

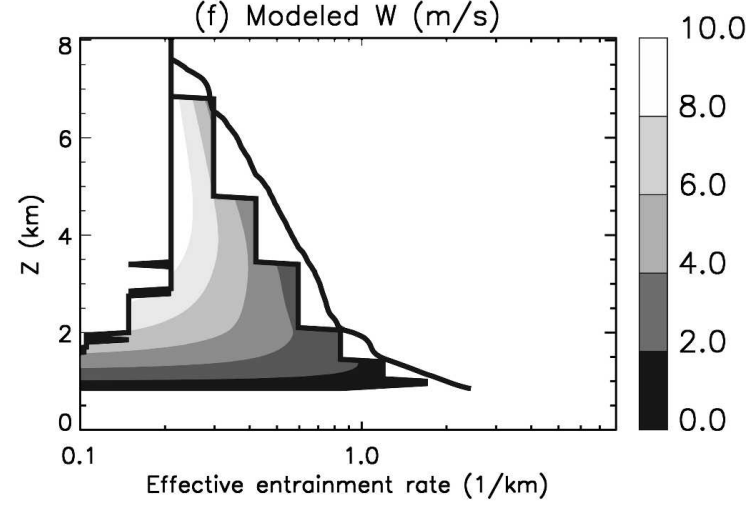

(h) Modeled condensate $(\mathrm{g} / \mathrm{kg})$

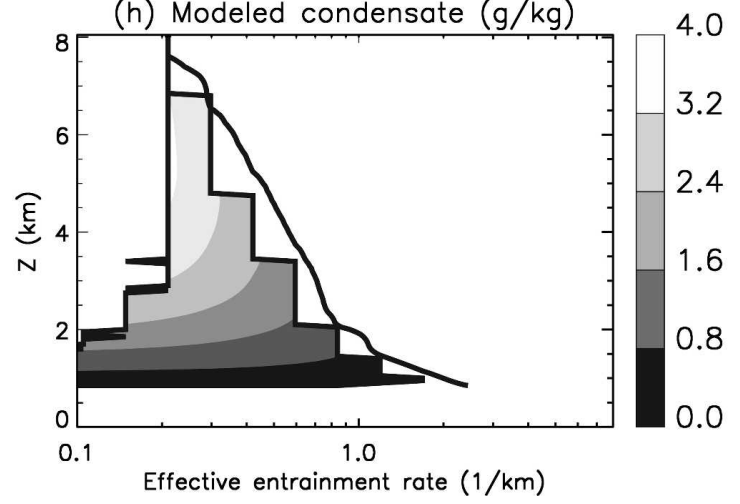

FIG. 12. (a)-(f) Same as Fig. 10, except for the deep cumulus regime. Also presented are the results for total condensate loading (both precipitating and nonprecipitating) from the (g) CRM simulation and (h) from the entraining plume model. 


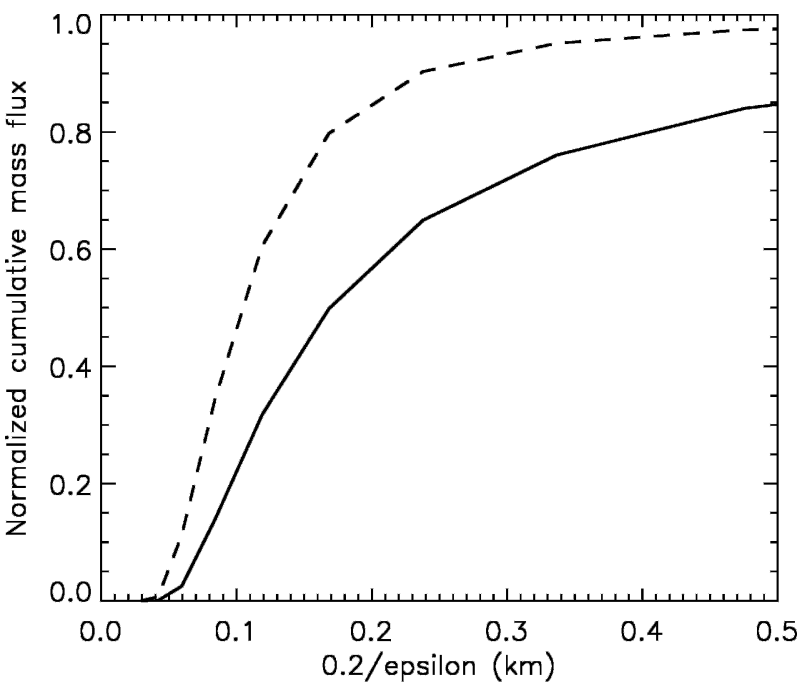

FIG. 13. Normalized cumulative mass flux at $1 \mathrm{~km}$ as a function of $0.2 / \varepsilon$ for the shallow (dashed) and deep (solid) cumulus regimes.

question is what determines the partitioning of cloudbase mass flux between the plume types. Similarity arguments and classical laboratory experiments on thermals suggest that the fractional entrainment rate of a simple entraining plume should be proportional to the inverse of its radius. Our "plumes" are better interpreted as composites of subcloud drafts rather than entire clouds, but one might still hypothesize that the distribution of effective cloud-base entrainment rates $\varepsilon$ is related to the distribution of cloud sizes.

To test this idea, we associated an entraining radius $R_{\varepsilon}=0.2 / \varepsilon$ with each entrainment rate $\varepsilon$. Figure 13 plots the normalized cumulative mass flux at $z=1 \mathrm{~km}$ in plumes with entrainment rate greater than $\varepsilon$ versus $R_{\varepsilon}$, for the shallow and deep regimes. The $1-\mathrm{km}$ analysis height is chosen because the finite spread of cloud base thermodynamic properties corrupts our analysis of the partitioning of mass flux versus $\varepsilon$ closer to cloud base. The shallow regime has more mass flux partitioned into low $R_{\varepsilon}$, highly entraining plume types.

Figure 13 can be compared with the simulated distribution of cloud sizes. We group cloudy grid points at a given height that connect with an edge, identify them as one cloud, and calculate the area and perimeter of each cloud. Figure 14 plots the cumulative cloud mass flux as a function of the equivalent cloud radius $r=2 \times$ area/ perimeter (so defined to account for the irregular shape of the clouds) at $1 \mathrm{~km}$. The cloud-size distributions are similar at the cloud base and at $1-\mathrm{km}$ altitude. There are more large clouds in the deep cumulus regime, as also noted by Grabowski et al. (2006). Inspection of sample cloud fields shows that clouds in the shallow cumulus

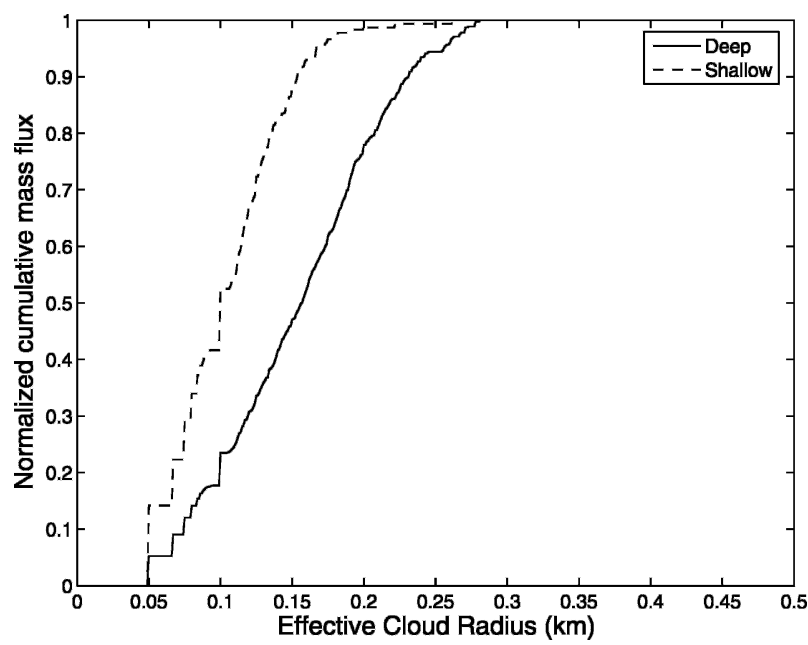

FIG. 14. Normalized cumulative mass flux at $1 \mathrm{~km}$ by clouds with different effective cloud radii for the shallow (dashed) and deep (solid) cumulus regimes.

regime tend to be randomly located, while clouds in the deep cumulus regime tend to lie on the edges of cold pools, as noted by Tompkins (2001). This mesoscale organization appears to induce broader boundary layer updrafts that produce larger cloud bases.

Figures 13 and 14 provide some support for the hypothesis that deep cumuli have somewhat lower entrainment rates because they are larger, and qualitatively suggest that the concept of an exponential or lognormal distribution of plume radii that control the partitioning of mass flux between entrainment rates, and whose mean value is controlled by the subcloud layer depth and some measure of mesoscale heterogeneity, might be adequate for parameterization purposes. This conclusion, however, must be viewed as tentative, and requires further testing. We note, for example, that the distributions in Fig. 13, tend to be broader, with long tails of low entrainment rate plumes (large $R_{\varepsilon}$ ) not seen in the cloud-size distribution (Fig. 14). Besides the difficulty in calculating cloud-size distribution due to the irregular shapes of clouds, this discrepancy may also reflect in part the variety of mixtures seen within individual cumuli of any size, and may also reflect aliasing of the finite spread in the thermodynamic properties of cloud base air into a wider range of apparent $\varepsilon$ at $1 \mathrm{~km}$, even though this is several hundred meters above the cloud base. Moreover, we have adopted a simple model with a constant entrainment rate for each plume in this paper. In a recent study of shallow cumulus convection that includes a feedback between entrainment rate and vertical velocity, the distributions of thermodynamic properties at the cloud 
base, while narrow, were sufficient to explain the variability in the cumulus clouds (Neggers et al. 2002), implying no role for the cloud sizes. Further investigation of this issue is clearly necessary.

\section{Summary}

The present paper is an attempt to use highresolution cloud-resolving simulations to improve parameterizations of cumulus convection in large-scale models. While alternative CRM-based approaches to large-scale modeling are being introduced to eliminate the need for traditional cumulus parameterizations for some problems (Arakawa 2004; Grabowski 2001; Kuang et al. 2005), most global weather and climate models continue to rely on cumulus parameterizations. Furthermore, such parameterizations are intrinsically valuable as integrated and testable summaries of our conceptual understanding of cumulus convection (with the caveat that they must ultimately be integrated into a broader modeling system with strong constraints due to discretization and imperfect representations of other physical processes).

We have analyzed, from a mass-flux scheme perspective, cloud and transport statistics, and their evolution during an idealized numerical experiment of a transition from shallow to deep cumulus convection. We focused on the initial properties of convective updrafts at the cloud base, how the mass flux and the bulk properties of the convective updrafts evolve with height, and the cloud-base mass-flux closure and partitioning problems. The present simulation does not have intense precipitation, even after convection reaches the upper troposphere. The results showed the following:

1) The initial thermodynamic properties of the updrafts at the cloud base have tight distributions for both shallow and deep convection, suggesting that a single representation in the cumulus scheme may be sufficient.

2) A model with a spectrum of entraining plumes appears to reproduce most features of cloudy updrafts throughout the CRM simulation, except the decrease in mass flux as the "plumes" approach their levels of zero buoyancy. A potential remedy to this deficiency is to include detrainment as in, for example, a buoyancy-sorting scheme. As the present simulation never has very intense precipitation, and downdrafts are rather weak, we have focused our attention on the updrafts. Further extension to deep convection with strong precipitation and downdrafts is needed.

3) Contrary to the assumption made in many cumulus schemes, nearly undiluted air parcels are too infrequent to be relevant to any stage of the simulated convection.

4) It is suggested that the spectrum of apparent entrainment rates may be tied to the basal cloud-size spectrum. In precipitating convection, the organized circulations of cold pools seem to create clouds with larger-sized bases and correspondingly smaller lateral entrainment rates.

5) The time evolution of CAPE, CIN, TKE, and the cloud-base mass flux does not support a mass-flux closure based solely on CAPE. Our results are in agreement with a CIN-based closure tying mass flux to $\mathrm{TKE}^{1 / 2} \exp (-k \mathrm{CIN} / \mathrm{TKE})$.

6) The general similarity in the ensemble characteristics of shallow and deep convection and the continuous evolution of the thermodynamic structure during the transition provide justification for developing a unified cumulus parameterization that encompasses both shallow and deep convection.

Finally, the statistical methodologies that we used in this paper, in particular that described in section $3 \mathrm{c}$, allow rather direct comparison between CRM outputs and cumulus schemes, and are suggested as an attractive way for analyzing CRM experiments that are aimed at improving cumulus parameterizations.

Acknowledgments. This research was supported by NASA Grant NAG5S-10624 and a NOAA Climate and Global Change postdoctoral fellowship awarded to Kuang. We thank Marat Khairoutinov for providing the SAM model, Peter Blossey for providing the code for the cloud-size distribution analysis, Chris Walker for help with the graphics and comments on the manuscript, and three reviewers for constructive comments that helped improve the presentation of this paper.

\section{REFERENCES}

Arakawa, A., 2004: The cumulus parameterization problem: Past, present, and future. J. Climate, 17, 2493-2525.

_ , and W. H. Schubert, 1974: Interaction of a cumulus cloud ensemble with large-scale environment.1. J. Atmos. Sci., 31, 674-701.

Bechtold, P., E. Bazile, F. Guichard, P. Mascart, and E. Richard, 2001: A mass-flux convection scheme for regional and global models. Quart. J. Roy. Meteor. Soc., 127, 869-886.

Bretherton, C. S., and P. K. Smolarkiewicz, 1989: Gravity waves, compensating subsidence and detrainment around cumulus clouds. J. Atmos. Sci., 46, 740-759.

_ J. R. McCaa, and H. Grenier, 2004: A new parameterization for shallow cumulus convection and its application to marine subtropical cloud-topped boundary layers. Part I: Description and 1D results. Mon. Wea. Rev., 132, 864-882.

Carpenter, R. L., K. K. Droegemeier, and A. M. Blyth, 1998: En- 
trainment and detrainment in numerically simulated cumulus congestus clouds. Part I: General results. J. Atmos. Sci., 55, 3417-3432.

Cohen, C., 2000: A quantitative investigation of entrainment and detrainment in numerically simulated cumulonimbus clouds. J. Atmos. Sci., 57, 1657-1674.

Derbyshire, S. H., I. Beau, P. Bechtold, J.-Y. Grandpeix, J.-M. Piriou, J.-L. Redelsperger, and P. M. M. Soares, 2004: Sensitivity of moist convection to environmental humidity. Quart. J. Roy. Meteor. Soc., 130, 3055-3080.

Emanuel, K. A., 1991: A scheme for representing cumulus convection in large-scale models. J. Atmos. Sci., 48, 2313-2335.

Fritsch, J. M., and C. F. Chappell, 1980: Numerical prediction of convectively driven mesoscale pressure systems. Part I: Convective parameterization. J. Atmos. Sci., 37, 1722-1733.

Grabowski, W. W., 2001: Coupling cloud processes with the largescale dynamics using the Cloud-Resolving Convection Parameterization (CRCP). J. Atmos. Sci., 58, 978-997.

— , and Coauthors, 2006: Daytime convective development over land: A model intercomparison based on LBA observations. Quart. J. Roy. Meteor. Soc., 132, 317-344.

Holland, J. Z., and E. M. Rasmusson, 1973: Measurements of atmospheric mass, energy, and momentum budgets over a 500kilometer square of tropical ocean. Mon. Wea. Rev., 101, 4455.

Igau, R. C., M. A. LeMone, and D. Y. Wei, 1999: Updraft and downdraft cores in TOGA COARE: Why so many buoyant downdraft cores? J. Atmos. Sci., 56, 2232-2245.

Khairoutdinov, M. F., and D. A. Randall, 2003: Cloud resolving modeling of the ARM summer 1997 IOP: Model formulation, results, uncertainties, and sensitivities. J. Atmos. Sci., 60, 607625

Kuang, Z. M., P. N. Blossey, and C. S. Bretherton, 2005: A new approach for 3D cloud-resolving simulations of large-scale atmospheric circulation. Geophys. Res. Lett., 32, L02809, doi:10.1029/2004GL021024.

Lin, C. C., 1999: Some bulk properties of cumulus ensembles simulated by a cloud-resolving model. Part I: Cloud root properties. J. Atmos. Sci., 56, 3724-3735.
—, and A. Arakawa, 1997: The macroscopic entrainment processes of simulated cumulus ensemble. Part II: Testing the entraining-plume model. J. Atmos. Sci., 54, 1044-1053.

Mapes, B. E., 2000: Convective inhibition, subgrid-scale triggering energy, and stratiform instability in a toy tropical wave model. J. Atmos. Sci., 57, 1515-1535.

Neggers, R. A. J., A. P. Siebesma, and H. J. J. Jonker, 2002: A multiparcel model for shallow cumulus convection. J. Atmos. Sci., 59, 1655-1668.

,,-- G. Lenderink, and A. A. M. Holtslag, 2004: An evaluation of mass flux closures for diurnal cycles of shallow cumulus. Mon. Wea. Rev., 132, 2525-2538.

Nitta, T., and S. Esbensen, 1974: Heat and moisture budget analyses using BOMEX data. Mon. Wea. Rev., 102, 17-28.

Pennell, W. T., and M. A. Lemone, 1974: An experimental study of turbulence structure in the fair-weather trade wind boundary layer. J. Atmos. Sci., 31, 1308-1323.

Raymond, D. J., and A. M. Blyth, 1986: A stochastic mixing model for nonprecipitating cumulus clouds. J. Atmos. Sci., 43, 2708-2718.

Siebesma, A. P., and J. W. M. Cuijpers, 1995: Evaluation of parametric assumptions for shallow cumulus convection. J. Atmos. Sci., 52, 650-666.

—- and Coauthors, 2003: A large eddy simulation intercomparison study of shallow cumulus convection. J. Atmos. Sci., 60, 1201-1219.

Tompkins, A. M., 2001: Organization of tropical convection in low vertical wind shears: The role of cold pools. J. Atmos. Sci., 58, 1650-1672.

Zhang, G. J., and N. A. McFarlane, 1995: Sensitivity of climate simulations to the parameterization of cumulus convection in the Canadian Climate Center general-circulation model. Atmos.-Ocean, 33, 407-446.

Zhao, M., and P. H. Austin, 2005a: Life cycle of numerically simulated shallow cumulus clouds. Part I: Transport. J. Atmos. Sci., 62, 1269-1290.

_ , and — 2005b: Life cycle of numerically simulated shallow cumulus clouds. Part II: Mixing dynamics. J. Atmos. Sci., 62, 1291-1310. 